diese Einheiten im Zuge der Umstrukturierung des Konzerns und bei ihren täglichen Geschäften eine Vielzahl anderer Sorgen haben, verzögert sich der Fortgang des Projekts. Statt ursprünglich 4 Monate sind nun etwa 8 Monate für die zweite Phase angedacht. Sowohl Hoechst als auch das Öko-Institut können mit dieser Verzögerung leben. Es ist wichtiger, ein schlüssiges, anwendbares System der Umsetzung des Leitbildes der nachhaltigen Entwicklung zu erarbeiten, als exakte Termine einzuhalten.

Die Erfahrungen aus der alltäglichen Arbeit zeigen, daß der Blick von außen wichtig ist. Er hilft vor allem dabei, den Blickwinkel zu erweitern: Während man in Hoechst über einzelne Produkte und Anlagen Informationen zusammenträgt, zeigt das Öko-Institut den Systemcharakter auf. Dabei darf nicht vergessen werden, daß es nicht um eine umfassende Operationalisierung des Leitbildes der nachhaltigen Entwicklung gehen kann. Jede Menge Forscher arbeiten im Auftrag einer Vielzahl von Auftraggebern an diesem Thema. Nicht umsonst wurde in Rio 1992 die Erarbeitung von Indikatoren für eine nachhaltige Entwicklung für besonders dringlich erachtet. Statt einer umfassenden Operationalisierung geht es darum, den Blick der operativen Einheiten für die Anforderungen, die mit dem Leitbild der nachhaltigen Entwicklung verbunden ist, zu schärfen.

Im Augenblick überwiegen noch immer die vielen Fragen und Probleme. Antworten und Lösungsansätze tauchen auf und werden auf ihre Tragfähigkeit hin abgeklopft. Patentlösungen werden nicht herauskommen. Wir haben sie aber auch nicht erwartet.

\section{Die Autoren}

Der Arrikel wurde in Teilen gemeinsum geschrieben. Die Abschnitte, die von einem Autor allein geschrieben wurden, sind im folgenden nach dem Namen aufgeführt.

Dr. Wolfgang Brühl ist Leiter Wirtschafts- und Marktanalysen, Hoechst AG $I_{\text {, Einbindung der Kooperation }}$ in die Veränderungen bei Hoechst"), Frank Ebinger ist wissenschaftlicher Mitarbeiter am Öko-Institut, Projekte "Green TV" und "Hoechst nachhaltig" I, Einordnung in die aktuelle Diskussion") und Christoph Ewen ist wissenschaftlicher Koordinator am ÖkoInstitut und Projektleiter bei "Hoechst nachhaltig" (Einführung, "Was passiert konkret",).

Kontakt: Hoechst AG, Wirtschafts- und Markianalysen, 65926 Frankfurt; Öko-Institut e.V., Postfach 6226, 79038 Freiburg

Zunehmende Bedeutung von Kooperation für Innovationsergebnisse

\title{
Netzwerke und Innovation
}

\section{Es ist inzwischen allgemein anerkannt, daß die Entstehung von Innovationen in der Regel mit einem bedeutenden Ausmaß an Interaktion und Arbeitsteilung zwischen verschiedenen Organisationen verbunden ist. Empirische Studien bele- gen eindrucksvoll, daß sowohl die Verbreitung als auch das Ausmaß dieser Arbeitsteilung während der letzten Jahrzehnte deutlich zugenommen hat.}

$\mathrm{I}$

Von Michael Fritsch nfolge der stetig steigenden Dynamik und Intensität des sich zunehmend globalisierenden Wettbewerbs ist die Innovationsfähigkeit eines Unternehmens zu einem entscheidenden Wettbewerbsfaktor geworden. Dies gilt insbesondere für eine Hochlohnnation wie die Bundesrepublik Deutschland, die gegenüber Niedriglohnländern Kostennachteile bei der Produktion von standardisierter Massenware aufweist. Durch die Kooperation von Unternehmen mit anderen Institutionen (Unternehmen, Forschungseinrichtungen, Universitäten etc.) bzw. durch Kooperationsnetzwerke läßt sich der Innovationsprozeß deutlich effizienter gestalten. Empirische Analysen belegen eindrucksvoll, daß Innovationsprozesse in zunehmendem Maße durch Arbeitsteilung bzw. Kooperation gekennzeichnet sind. Dies macht eine Ausweitung der analytischen Perspektive erforderlich: Nicht allein das einzelne innovierende Unternehmen, sondern das gesamte Netzwerk an Kooperationsbeziehungen ist entscheidend für die Innovationsergebnisse.

\section{Formen von Kooperation}

Man spricht im Zusammenhang mit arbeitsteiliger Innovation häufig von 'Kooperation', wobei dieser Begriff z.T. sehr unterschiedlich und dabei oft auch recht unscharf definiert ist. 'Kooperation' stellt eine spezielle Form von Arbeitsteilung dar und meint in der Regel solche Austauschbeziehungen, die in wesentlichem Ausmaß 'weiche' Elemente enthalten. Unter derartigen 'weichen' Elementen versteht man solche Vertragsbestandteile, in denen die Verpflichtungen der Vertragspartner - anders als bei 'normalen' Kaufverträgen - nur unvollständig spezifiziert sind. Entsprechend dieser Definition stellt der. Großteil der Formen arbeitsteiliger Innovation eine Kooperation dar. Denn Innovationstätigkeit beinhaltet ja im Kern die Generierung neuen Wissens, wobei das Resultat des entsprechenden Suchprozesses nicht genau vorhersehbar ist und deshalb auch das Ergebnis von Arbeitsteilung im Bereich von Forschung und Entwicklung (F\&E) im vorhinein nur ungenau vertraglich festgelegt werden kann. Aus der für 'weiche' Beziehungen charakteristischen unvollständigen Definition des Vertragsgegenstandes ergibt sich wiederum die Gefahr, daß sich ein Vertragspartner opportunistisch verhält und unklare Regelungen einseitig zu seinen Gunsten auslegt. Kooperation setzt daher ein gewisses Mindestmaß an Vertrauen der Kooperationspartner zueinander voraus.

Als ein System von Kooperationsbeziehungen können sogenannte 'Netzwerke' angesehen werden. Sie stellen komplexere Geflechte von Kooperationsbeziehungen zwischen mehr als zwei Partnern dar, wobei zwischen diesen Netzwerkpartnern nicht selten eine gemeinsame Interaktions'kultur' besteht, die sich in 'ungeschriebenen Regeln' niederschlägt. Die einem solchen Netzwerk angehörenden Firmen sind häufig in bestimmten Regionen konzentriert, was sich aus der Bedeutung von häufigen faceto-face-Kontakten für den Aufbau einer vertrauensvollen Zusammenarbeit ergibt.

Als wesentliche konkrete Formen der F\&EKooperation lassen sich unterscheiden: - Durchführung der gemeinsamen F\&EAktivitäten in einer separaten organisatorischen Einheit, die den Kooperationspartnern gemeinsam gehört (Joint Venture).

- Zielgerichtete Koordination der Aufgaben von rechtlich selbständigen Unternehmen im Rahmen bestimmter Projekte (F\&E Konsortium, Strategische Allianz) mit oder ohne (teilweiser) Zusammenlegung entsprechender Kapazitäten.

- Gemeinsame Nutzung von Laboratorien oder bestimmten Geräten (z.B. Meß- und Prïfeinrichtungen).

- Die Vereinbarung der gegenseitigen Erteilung gebuihrenfreier Lizenzen aus Patenten (,Lizenz- 
tausch“, „Patentpool“), die aus bestimmten F\&E-Aktivitäten resultieren.

- Das 'information trading' von Mitarbeitern miteinander konkurrierender Firmen, d.h. der informelle gegenseitige Austausch von Erfahrungen und Know-How.

- Zusammenarbeit (etwa in Arbeitskreisen, Verbänden) in den Bereichen Gütesicherung, Standardisierung und Normung.

Die externe Vertragsforschung, die Inanspruchnahme von innovationsorientierten Dienstleistungen (z.B. Labor- oder Ingenieursleistungen) sowie der Kauf oder Verkauf von Lizenzen sind wohl nur als F\&E-Kooperationen im weiteren Sinne anzusehen, da sich hier die Leistungen der Vertragspartner im vorhinein relativ genau definieren lassen.

Differenziert man nach der Stellung der Kooperationspartner im Marktprozeß, so sind vertikale F\&E-Kooperationen, also die Zusammenarbeit zwischen Abnehmern und $\mathrm{Zu}$ lieferern, mit Abstand am weitesten verbreitet, wobei auch Universitäten und öffentliche Forschungseinrichtungen als Zulieferer im weiteren Sinne aufgefaßt werden können. F\&EKooperationen zwischen Wettbewerbern (horizontale Kooperation) finden demgegenüber deutlich seltener statt. Da ein wesentliches Ziel der horizontalen Innovations-Kooperation auch in der Verhaltensabstimmung und damit im Aufbau oder der Verstärkung von Marktmacht bestehen kann, sind bestimmte Formen einer solchen Zusammenarbeit von Wettbewerbern im F\&E-Bereich wirtschaftspolitisch nicht unbedenklich.

\section{- Vorteile der Innovations- kooperation}

Die Vorteile bzw. Motive der Innovationskooperation von Unternehmen mit anderen externen Akteuren sind vielfältig. Hierbei handelt es sich im wesentlichen um:

- Überwindung von unternehmensinternen Engpässen, was dann die Durchführung von Innovationsprojekten ermöglicht, die einzeln nicht realisiert werden könnten.

- Aufteilung des F\&E-Aufwandes auf diejenigen Unternehmen, die von den Ergebnissen profitieren. Dieses Motiv ist insbesondere dann von Bedeutung, wenn sich die Verbreitung und unentgeltliche Nutzung der F\&E-Ergebnisse nur schwer verhindern läßt.

- Aneignung von externem Wissen und Stimulierung von Lerneffekten.
- Aufteilung des Innovationsrisikos auf die Kooperationspartner.

- Vermeidung von Doppelarbeit.

- Hilfe beim Einstieg in ein für das betreffende Unternehmen völlig neues Technologiegebiet.

- Beschleunigung der Entwicklung und damit Verkürzung der ,time to market“.

- Ausschöpfung von Größen- und Spezialisierungsvorteilen sowie

- Erleichterung bei der Entwicklung, Einfiihrung und Durchsetzung bestimmter technischer Standards.

Speziell die kooperative Zusammenarbeit mit Pilotanwendern (,Lead-Usern“) kann den innovierenden Unternehmen dabei helfen, die Produktanforderungen marktgerecht $\mathrm{zu}$ definieren und mögliche Implementationsprobleme frühzeitig zu erkennen. Die Kooperation mit den Zulieferern ermöglicht die Sensibilisierung dieser vorgelagerten Produzenten für zukünftige Anforderungen an ihre Produkte und kann sich von der Zusammenarbeit bei speziellen Problemlösungen bis hin zur Entwicklung kompletter Systementwicklung erstrecken.

\section{Forschungs- und Entwicklungskooperation}

Ein wesentliches Problem der F\&E-Kooperation wurde bereits eingangs angesprochen: Sie setzt ein gewisses Vertrauen darin voraus, daß der Vertragspartner unklare Regelungen nicht einseitig zu seinen Gunsten auslegt. Ein weiteres Problem stellt die Gefahr eines unkontrollierten Know-How-Abflusses dar. Untersuchungen von F\&E-Kooperationen kleiner und mittelgroßer Unternehmen in Deutschland haben ergeben, daß ein wesentlicher Engpaß für das Zustandekommen einer Zusammenarbeit in der Identifikation eines geeigneten Kooperationspartners und der Abschätzung seiner Leistungsfähigkeit besteht. Als Gründe, die gegen eine F\&E-Kooperationssprechen, werden vor allem die notwendige Offenlegung von Innovations- und Produktstrategien gegenüber dem Partner, das Bestreben nach Bewahrung von technologischer Eigenständigkeit und technologischer Kernkompetenz, die im Rahmen der Kooperation erforderliche Preisgabe bisheriger F\&E-Ergebnisse sowie die Höhe des Koordinationsaufwandes genannt.

Vielfach wird betont, daß kooperative Arbeitsteilung in besonderem Maße Lerneffekte stimuliert und $\mathrm{zu}$ einer relativ schnellen Übernahme von Innovationen fuihrt. Dies wird insbesondere auf die in 'weichen' Beziehungen in der Regel relativ 'offenen' (d.h. nicht erschöpfend definierten) Schnittstellen zwischen den Kooperationspartnern zurückgeführt. So sind F\&EKooperationen häufig durch ein hohes Maß an gemeinsamen Problemlösungen gekennzeichnet, was nichts anderes als kooperatives Lernen bedeutet.

Bisher vorliegende empirische Untersuchungen deuten darauf hin, daß F\&E-Kooperation wesentlich zum Innovations- und damit letztlich zum Unternehmenserfolg beiträgt. Probleme der Meßbarkeit der relevanten Variablen legen allerdings einen kritischen Umgang mit den bisherigen Ergebnissen nahe. Sowohl die Intensität von Kooperationsbeziehungen als auch der Innovationserfolg entziehen sich nämlich der exakten Quantifizierung und können nur mit relativ groben Indikatoren erfaßt werden. Probleme bei der Erfassung des Zusammenhangs zwischen F\&E-Kooperation und Unternehmenserfolg ergeben sich auch daraus, daß der Großteil der F\&E-Kooperation in einer vorwettbewerblichen Phase stattfindet, also zu einer Zeit, in der sich die betreffenden Produkte noch nicht auf dem Markt befinden. Die oftmals erhebliche Zeitverzögerung, mit der sich die gemeinsam entwickelten Innovationen im kommerziellen Erfolg der Unternehmen niederschlagen, machen die Zuordnung von Ursachen und Wirkungen meist sehr schwer.

\section{Trend zu kooperativen Innovationsprozessen}

Diverse empirische Untersuchungen belegen eine zunehmende Bedeutung von Arbeitsteilung im Innovationsprozeß. So ergibt eine Untersuchung für Deutschland, daß der Anteil der F\&Etreibenden Unternehmen, die mindestens eine F\&E-Kooperationsbeziehung unterhalten, von weniger als 10\% im Jahr 1971 auf knapp 50\% im Jahre 1992 angestiegen ist. Berechnungen des Stifterverbandes Wissenschaftsstatistik weisen einen erheblichen Anstieg desjenigen Anteils des F\&E-Budgets privater Unternehmen aus, das für Externe aufgewendet wird (3,1\% im Jahr 1975; 8,4\% im Jahr 1987). Entsprechend haben sich etwa die Drittmittel der Hochschulen aus dem Sektor der privaten Unternehmen zwischen 1980 und 1985 mehr als verdoppelt.

In der Literatur werden verschiedene Faktoren diskutiert, die verantwortlich für den Trend $\mathrm{zu}$ zunehmender Arbeitsteilung im Innovationprozeß sein könnten: 
- Zum einen wird die steigende Bedeutung der Technologiefusion im Innovationsprozeß hervorgehoben. Im Rahmen einer solchen Technologiefusion werden Innovationen durch die Verbindung mehrerer, vorher separater Technikfelder generiert. Beispiele wären etwa die Optoelektronik, die aus der Verbindung von Optik und Elektronik hervorging (z.B. Faseroptik-Kommunikationssysteme) sowie die Mechatronik, die Verschmelzung von Mechanik und Elektronik, welche die WerkzeugmaschinenBranche nachhaltig veränderte.

- Zweitens wird der zunehmende 'systemische' Charakter von Innovationen hervorgehoben, wobei der Begriff 'systemisch' eine relativ hohe Komplexität meint, die insbesondere eine Interaktion verschiedener Akteure (etwa Zulieferer und Abnehmer) erfordert.

- Eine dritte Erklärung beruht auf der Hypothese, daß sich der Innovationsprozeß selbst während der letzten Jahrzehnte wesentlich verändert hat. Demnach sind F\&E-Aktivitäten immer weniger durch ein empiristisches trial-and-errorVorgehen, sondern mehr und mehr durch den Versuch eines grundlegenden Verständnisses der relevanten Zusammenhänge gekennzeichnet. Während das im trial-and-error Verfahren generierte Wissen nur schwer auf andere Zusammenhänge übertragen werden kann, erfordert die stärker wissenschaftlich geprägte Suche nach grundlegenden Erklärungen eher abstrakte und damit vielfältiger verwendbare Informationen, wobei die Generierung und Übermittlung solcher Informationen durch Fortschritte in der Computer- und Übertragungstechnik beginstigt wird. Ist neues, innovationsrelevantes Wissen nun vielfältiger verwendbar und leichter übertragbar, dann folgt daraus, daß der Innovationsprozeß leichter und lohnender arbeitsteilig gestaltet werden kann. Dies impliziert eine wachsende Bedeutung von Märkten für innovationsrelevante Informationen sowie zunehmende Relevanz rechtlicher Regelungen zum Schutz geistigen Eigentums.

\section{Schlubfolgerungen und weitere Fragen}

Ohne Zweifel stellt Kooperation einen außerordentlich wichtigen Aspekt von Innovationsprozessen dar. Die große und in letzter Zeit zunehmende Bedeutung von F\&E-Kooperationen impliziert, daß es sowohl für die wissenschaftliche Analyse aber auch für eine Politik der Innovationsförderung nicht ausreicht, allein auf den einzelnen Innovator abzustellen. Um den Innovationspro- zeß in seiner Arbeitsteiligkeit zu erfassen, muß jeweils das gesamte relevante 'Netzwerk' mit einbezogen werden. So geht denn auch die Innovationsforschung zunehmend dazu über, nationale, sektorale bzw. regionale Innovationssysteme zu betrachten. Aus der Sicht einer Volkswirtschaft oder Region fragt sich dabei insbesondere:

- Wie läßt sich das Innovationssystem so stimulieren, daß die Wirtschaft eines bestimmten Landes bzw. einer bestimmten Region besser (intensiver, schneller) an dem anderswo generierten Wissen teilhaben kann?

- Inwiefern kann die Innovationsleistung durch Verbesserung der Arbeitsteilung gesteigert werden?

- Auf welche Weise läßt sich die 'Vernetzung' der F\&E-Aktivitäten verbessern?

Ein zentraler Ansatzpunkt für weitere Forschungen kann in den Rahmenbedingungen und den Engpässen der arbeitsteiligen Innovation gesehen werden. Diesbezügliche Fragen wären etwa: - Wie kann der Schutz geistigen Eigentums verbessert werden?

- Welche Qualifikationen (z.B. Kooperationsfähigkeit, Innovationsmanagement) sind für den Erfolg arbeitsteiliger Innovationsprozesse notwendig und wie können solche Qualifikationen vermittelt werden?

- Welche Bedeutung kommt der institutionellen Infrastruktur (Universitäten, öffentliche Forschungsinstitute, Transfereinrichtungen etc.) für arbeitsteilige Innovationsprozesse $\mathrm{zu}$ und wie läßt sich deren Beitrag zum Innovationsprozeß steigern?

Von großem Interesse wäre auch, mehr über die Bedeutung der einzelnen Akteurs-Gruppen im arbeitsteiligen Innovationsprozeß und über den Einfluß des räumlichen Umfeldes zu erfahren.

\section{Anmerkung}

Zu einer ausführlichen Darstellung und mit Quellenangaben siehe Michael Fritsch: Arbeitsteilige Innovation - Ein Überblick über nevere Forschungsergebnisse, in: Dieter Sauer und Hartmut Hirsch-Kreinsen (Hrsg.): Zwischenbetriebliche Arbeitsteilung und Kooperation, Campus, Frankfurt/New York 1996, S. 15-47.

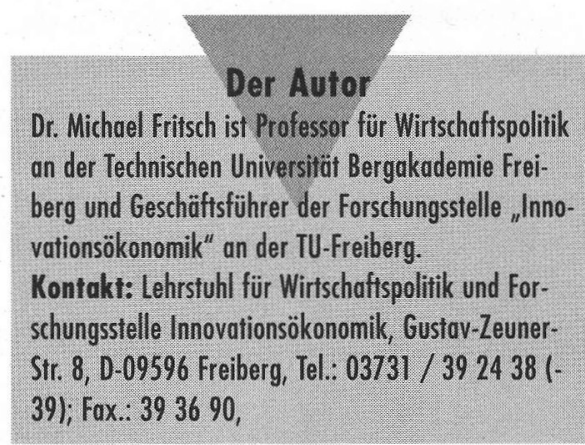

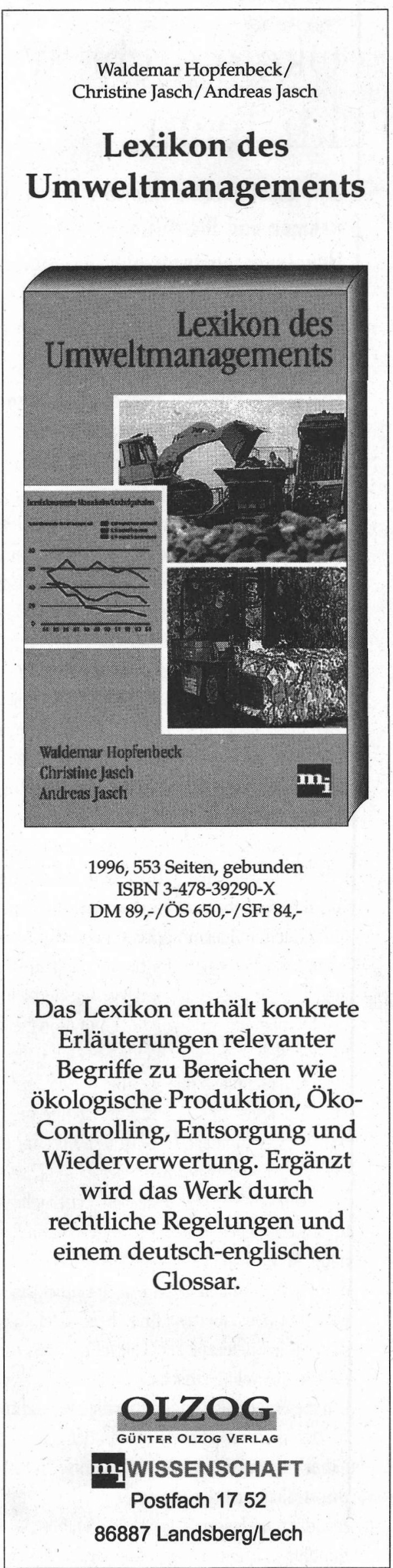


(c) 20I0 Authors; licensee IÖW and oekom verlag. This is an article distributed under the terms of the Creative Commons Attribution Non-Commercial No Derivates License (http://creativecommons.org/licenses/by-nc-nd/3.o/), which permits unrestricted use, distribution, and reproduction in any medium, provided the original work is properly cited. 